\title{
The effects of modified brief pulse bilateral electroconvulsive therapy (ECT) on disease profile and cognitive functions in patients of Depression, Mania and Schizophrenia
}

\author{
Dr Gaurav Singh, Dr Ritambhara Mehta, Dr Nilima Shah \\ E 2/7 Professor bungalow, New Civil Hospital Campus, Surat
}

\begin{abstract}
:
Title: The effects of modified brief pulse bilateral electroconvulsive therapy (ECT) on disease profile and cognitive functions in patients of Depression, Mania and Schizophrenia.

Aims \& Objectives:

In the patients of Depression, mania and Schizophrenia treated with modified brief pulse bilateral ECT, to assess

1. The type and amount of cognitive deficits pre and post-ECT.

2. The change in disease severity post-ECT.

\section{Materials and Methods:}

Consecutive patients with DSM-IV TR diagnosis of Bipolar Mania, Depressive Disorder(Major Depressive Disorder and Bipolar Depression) or Schizophrenia admitted and posted for modified brief pulse bilateral ECT were enrolled. Those who received ECT in past 6 months, were uncooperative or unwilling to consent were excluded. The scales below were administered before ECT, within 1 day of $1^{\text {st }}$ and last ECT, after 1 month of last ECT.

Tests/Scales used: For severity (as per disease)

1. PANSS (Positive and Negative Syndrome Scale)

2. HDRS (Hamilton Depression Rating Scale)

3. YMRS (Young Mania Rating Scale)

For cognitive functions:

1. (ACE - R) Addenbrook's cognitive rating scale

2. (MMMSE) Modified mini mental state examination

3. PGI memory test

Paired t test was used for significance detection.

Results \& Conclusions:

Patients enrolled (MDD: 23, Bipolar mania 22, Schizophrenia: 42) revealed:

1. ECT has acute effects on cognition that is evident by changes in cognitive profile of participants just after ECT. Cognitive decline caused by ECT is reversible.

2. ECT is effective in reducing disease severity in all 3 disorders.
\end{abstract}

\section{Introduction}

Electroconvulsive therapy is being used as a therapeutic procedure in treatment of psychiatric illnesses since last 60 years. It is most commonly used in treatment of Bipolar Mood Disorder, Major Depressive Disorder and Schizophrenia spectrum disorders. Even after the advent of newer effective medication Electroconvulsive therapy remains a widely used procedure throughout the world. It is generally considered to be useful in resistant cases as well as to provide quick relief from the psychopathology.

Despite its widespread use there has always been controversy over its effectiveness and side effects profile specially related to cognition. Some studies have concluded ECT to be no better than placebo and many have emphasized on the detrimental effects on cognition exceeding the benefit if any. Lack of adequate knowledge about the mechanism of action of Electroconvulsive therapy further complicates the picture. There is also a lack of clear consensus regarding efficacy and adverse effects of Electroconvulsive therapy in the psychiatry community.

This study is done to determine the degree of efficacy of Electroconvulsive therapy in patients of mania, depression and schizophrenia disorders by using appropriate illness related scales and to evaluate the effects of this therapy on cognition by using comprehensive battery of cognitive testing. 


\section{Aims \& Objectives}

A prospective comparative outcome study:

1. To assess the changes in disease severity in patients of MANIA, DEPRESSION and SCHIZOPHRENIA who are given ECT during the course of their hospital admission.

2. To assess the nature and quantity of cognitive changes in patients of MANIA, DEPRESSION and SCHIZOPHRENIA who are given ECT during the course of their admission.

\section{Review Of Literature}

\section{Controversy over long-term effects on general cognition}

In early 2007 first-ever large-scale study (347 subjects), Sackeim and colleagues found that at least some forms (namely bilateral application and outdated sine-wave currents) of ECT "routinely" lead to "adverse cognitive effects," including global cognitive deficits and memory loss, that persist for up to six months after treatment, suggesting that the induced deficits may be permanent. ${ }^{[4][5]}$

In July 2007, a second study was published concluding that ECT routinely leads to chronic, substantial cognitive deficits, and the findings were not limited to any particular forms of ECT. ${ }^{[6]}$ The study, led by psychiatrist Glenda MacQueen and colleagues, found that patients treated with ECT for bipolar disorder show marked deficits across multiple cognitive domains.

Breggin, chief editor of the journal Ethical Human Psychology and Psychiatry, is a leading critic of ECT who believes the procedure is neither safe nor effective. ${ }^{[5]}$

Six months after the publication of the Sackeim study ${ }^{[4]}$ documenting routine, long-term memory loss after ECT, prominent ECT researcher Max Fink published a review in the journal Psychosomatics concluding that patient complaints of memory loss after ECT are "rare" and should be "characterized as somatoform disorders, rather than as evidence of brain damage, thus warranting psychological treatment for such disorders. ${ }^{[7]}$

A number of reviews of the literature and other articles continue to characterize ECT as safe and effective..$^{[8[[9][10][11][12][13][14][15]}$ For example, in June 2009, Portuguese researchers published a review on the safety and efficacy of ECT in an article entitled, Electroconvulsive Therapy: Myths and Evidences. ${ }^{[8]}$ In their review, the researchers conclude that ECT is an "efficient, safe and even life saving treatment for several psychiatric disorders." In 2008, Yale researchers published a review on the safety and efficacy of ECT in elderly patients. ${ }^{[15]}$ According to the authors, "ECT is well established as a safe and effective treatment for several psychiatric disorders." And in a June 2009 article published in the Journal of ECT, Iranian researchers observe that, "Despite the wide consensus over the safety and efficacy of electroconvulsive therapy (ECT), it still faces negative publicity and unfavorable attitudes of patients and families." ${ }^{[14]}$

As seen from above discussion there is a lot of debate and controversy over cognitive effects of ECT.

\section{Materials And Methods}

Participants: All participants were given brief pulse bilateral ECT. Out of all participants who gave informed consent along with their relatives for participation in study 22 patients of mania, 23 patients of depressive disorder (20 major depressive disorder \& 3 bipolar depression), 42 patients of schizophrenia completed at least 3 steps of study and were taken for analysis. Of the above sample 20 patients of mania, 21 of depressive disorder and 35 of schizophrenia completed all four steps of study.

Duration of study: 2 years( january 2012 till December 2013)

Permitting authority and consent: study was approved by Human Resource Ethics Committee, Government Medical College, Surat, Gujarat, India.

Written informed consent of both parent and close relative was taken for enrollment in the study. Participants who were already posted on Electroconvulsive therapy were enrolled and no other additional intervention was done. 


\section{Steps of study:}

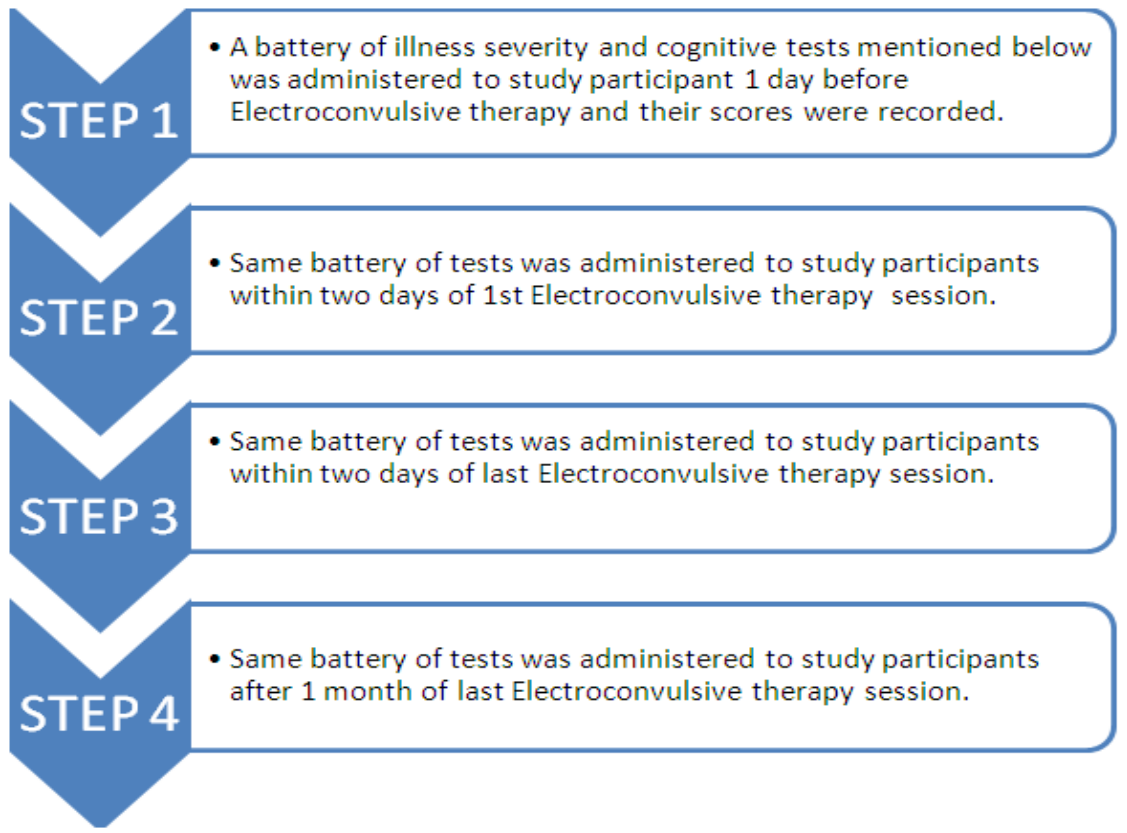

\section{Exclusion criterias:}

1. History of ECT within past 6 months

2. Patient uncooperative

\section{Tests/Scales administered:}

For disease severity:

4. HDRS (Hamilton Depression Rating Scale) was used to assess the severity of depression.

5. YMRS (Young Mania Rating Scale) was used to assess the severity of manic episode.

6. PANSS (Positive and Negative Syndrome Scale) was used to assess the severity of schizophrenia.

For cognitive functions evaluation

1. ACE - R (Addenbrook's cognitive rating scale).

2. MMMSE (Modified mini mental state examination).

3. PGI memory test.

Statistical analysis was done using Paired T Test with a p value of less then .05 as significant.

General Outline of Results \& Interpretation:

\section{Results}

In every scale that has been used in this study test of significance ( Paired $t$ test, level of significance <.05 ) is applied between the following:
1. $\mathrm{A} \& \mathrm{~B}$
2. $\mathrm{A} \& \mathrm{C}$
3. $\mathrm{A} \& \mathrm{D}$
4. $C \& D$
Where

A represents baseline score of the respective scale before ECT administration.

B represents scale score immediately after $1^{\text {st }} \mathrm{ECT}$ probably demonstrating the acute effects of ECT.

$\mathrm{C}$ represents scale score after last ECT presumed to demonstrate the effects of full course, long term effects of the initial ECTs and acute effects of last ECT .

D represents score after 1 month of ECT course administration probably reflecting the long term effects of ECT administration.

\section{For illness severity scale:}


Although decrease in severity scores can be caused either by ECT, concurrent medication or the natural course of illness or a combination of above factors; in this study ECT will be considered as significant contributing factor to the decrease in severity score.

\section{For cognitive scales:}

1. A decrease in cognitive score can be attributed to negative effects of ECT on brain or the worsening of illness during the course of ECT. In interpretation any decrease in cognitive score during the course of ECT is being considered as a negative effect of ECT.

2. An increase in cognitive score during the course of ECT will be considered to be due to decrease in illness severity as studies showing direct increase in cognition due to positive effects on brain by ECT are very few.

\section{Mania patients}

Participants: Total no: 22

20 completed all steps $\& 2$ didn't come for $4^{\text {th }}$ step

Demographic profile:

Average age: 31 years, Range: 22 years to 45 years

Sex: male: 15 , female: 7

Illness profile:

Average illness duration: 5 years, range: 1 month to 16 years

Average No of manic episode: $4^{\text {th }}$, range: 1 to 14

History of past ECT present: 8

Reason for posting to ECT: not responding to medication: 9, disruptive behavior: 7, hasten recovery: 4, past history of response to ECT: 2

Frequency of ECT administered: 3/week in 20, 2/week in 2

Average no of ECT given: 9 (highest 11, lowest 5)

Subjective complaint of memory impairment reported in patient: 4

\section{Depressive disorder patients}

\section{Participants: Total no: 23}

20 completed all steps. 3 didn't come for $4^{\text {th }}$ step

Diagnosis: Major Depressive Disorder (20), Bipolar 1 mood disorder depressed (3)

Demographic profile:

Average age: 31 years Range: 18 years to 51 years

Sex: male: 14 , female: 9

\section{Illness Profile:}

Average illness duration: 5 years, range: 1 month to 16 years

Average No of depressive episodes: 2.4, range: 1 to 6

History of past ECT present: 4

Reason for posting to ECT: not responding to medication: 12, suicidal ideations: 7 , hasten recovery: 3 , past history of response to ECT: 1

Frequency of ECT administered: 3/week in 18, 2/week in 5

Average no of ECT given: 6, highest 8, lowest 3

Subjective complaint of memory impairment reported in patient: 3

\section{Schizophrenia patients}

Participants: Total no: 42

34 completed all steps. 8 didn't come four $4^{\text {th }}$ step

Demographic profile:

Average age: 31.5 years Range: 19 years to 55 years

Sex: male: 31 , female: 11

Illness profile:

Average illness duration: 4.33 years, range: 6 month to 15 years

History of past ECT present: 8

Reason for posting to ECT: not responding to medication: 20, disruptive behaviour: 7 , hasten recovery: 10 , past history of response to ECT: 5

Frequency of ECT administered: 3/week in 32, 2/week in 10

Average no of ECT given: 5.6, highest 9, lowest 3

Subjective complaint of memory impairment reported in patient: 7 


\section{Illnesss severity scales scores:}

1. Analysis of Young Mania Rating Scale score $(\mathrm{N}=22)$

Table 1 (all tables provided at the end of article)

Interpretation of results: ECT resulted in decrease in psychopathology in mania in study participants as per YMRS scale.

2. Analysis of Hamilton Depression Rating Scale:

Table 2

Interpretation of results: ECT resulted in decrease in psychopathology in depression in study participants as per HDRS scale.

3. Analysis of PANSS rating scale:

Table 3

Interpretation of results: ECT resulted in decrease in severity of psychopathology in schizophrenia in study participants as per PANSS scale. The decrease was maximum in positive symptom and least in negative symptoms.

On comparing the overall results of illness severity scales it is seen that there was significant decrease in the scores in all 3 disorders after $1^{\text {st }}$ ECT, after last ECT and after one month of completion of last ECT.

Discussion: there was significant decrease in the scores in all 3 disorders after $1^{\text {st }}$ ECT, after last ECT and after one month of completion of last ECT. The effectiveness of ECT in these disorders in this study is in line with other similar significant studies ${ }^{[97][98] ~[99][97] ~[94] . ~ A l t h o u g h ~ s o m e ~ o f ~ t h e s e ~ s t u d i e s ~ h a v e ~ c o n s i d e r e d ~ o n l y ~}$ individual disorders with different illness severity scale yet they all showed significant decrease in psychopathology after ECT administration for example:

- The Leicester ECT trial ${ }^{[22]}$ showed significant greater improvement in participants when measured on the Montgomery-Asberg Schizophrenia Scale (MASS), the visual analogue global psychopathology scale and the depression scale.

- The study in Nepal carried out by Adhikari and Pradhan ${ }^{[18]}$ with comparison of 47 ECT receivers and 78 non receivers reported significant decrease in BPRS in ECT receiver as compared to non-receivers at discharge , 1st month, 6th month and 12th month; in YMRS at discharge, 1st month and at 12th month; in HAMD-M at discharge, at 1st month, at 6th month and at 12th month ; in GAF at discharge, at 6th month and at 12th month.

- The study done by Vineet Bharadwaj, Sandeep Grover \& Subho Chakrabarti ${ }^{[20]}$ in 32 Patients with severe depression while they were receiving ECT's reported gradual significant decline in MADRS scores.

- A meta analysis done by Iannis M Zervas, Christos Theleritis \& Costantin R Soldatos ${ }^{[21]}$ also reported ECT having significant benefit in reducing psychopathology in schizophrenia.

\section{Cognitive scales score:}

ACE-R scale scores

Table 4

MMMSE scale scores

Table 5

PGI Memory scale scores

Table 6

On comparison of cognitive profile in all three illnesses studied (mania, depression and schizophrenia) it was seen that:

1. Study participants of depression had the highest baseline mean cognitive scores while the study participants of mania had the lowest mean cognitive scores at baseline.

2. Study participants of mania showed cognitive decline on all 3 scales just after completion of first ECT, while study participants of depression showed cognitive decline only on PGI Memory scale after first ECT. Study participants of schizophrenia did not show cognitive decline on any of the scale after first ECT.

3. Study participants of mania showed cognitive impairment after completion of ECT course which was most marked in PGI Memory scale. Study participant of depression showed insignificant cognitive enhancement on ACE-R and MMMSE scales while PGI Memory scale showed insignificant cognitive decline. Study participants of schizophrenia showed significant cognitive enhancement on all three scales after completion of ECT course. 
4. After one month of last ECT study participants of all 3 illnesses showed improved cognitive scores on all 3 scales as compared to baseline cognitive score. Cognitive decline observed in study participants of mania also showed improved cognitive scores on all 3 scales as compared to baseline cognitive score.

\section{Discussion}

The finding of this study shows that ECT does not cause any long term cognitive impairment on the other hand it improves cognition by decreasing the illness severity.

- Similar findings were obtained in the study done by V. N. Bagadia. \& L. P. Shah ${ }^{[16]}$ in which no Post ECT cognitive deficit was observed on the test battery though some patients did complain of forgetfulness subjectively. .

- The study done by Neera Ghaziuddin \& Donna Laughrin ${ }^{[17]}$ also did not report any significant cognitive impairment during the course of ECT.

- The study done by $\mathrm{Ng} \mathrm{C}$ et $\mathrm{al}^{[19]}$ found that cognitive parameters found to be impaired during the first few days of ECT recovered over several months following the treatment. Therefore, there was no evidence of long-term damage to concentration, attention, verbal and visual memory, or verbal fluency.

- As most studies on cognition have concentrated upon memory part of cognition in this study on evaluating there was a decline in memory subset seen in mania and depression after last ECT which corresponded to finding of study of Sackeim et al. ${ }^{[4]}$ and Mcqueen et a ${ }^{[6]}$ but there was no permanent memory impairment as the memory impairment reverted back to baseline after 1 month. This is in contrast with these two studies. The possible reasons for this disparity can be the differences in sample size and test battery used.

- The results also show that illness related cognitive decline is a major confounding factor in evaluating cognitive effects of ECT.

\section{Conclusions}

1. Schizophrenia causes the most and depression causes the least illness related cognitive impairment.

2. ECT has acute effects on cognition that is evident by changes in cognitive profile of participants just after ECT. Cognitive decline caused by ECT is reversible as evident by increase in cognitive scores with subsequent return to baseline after 1 month of last ECT.

3. ECT is effective in reducing psychopathology in all 3 disorders which is further supported by the fact that even single ECT significantly brings down the psychopathology.

4. Decrease in psychopathology after completion of ECT is sustained over a period of one month.

To summarize: ECT course is beneficial in treatment of all 3 disorders studied and does not cause any long term cognitive deficit.

\section{Strengths And Limitations}

Strengths of this study:

- Three different disorders were compared together for effectiveness and cognitive evaluation after ECT.

- The scales used were administered by a single person hereby eliminating interrator bias. Every patient received Electroconvulsive therapy from the same machine and same protocol for ECT administration was followed in every patient which avoided any procedural bias.

- 3 different scales were used to test cognition which helped in identifying consistency of patient's responses.

Limitations of this study:

- Small sample size especially in mania and depression category.

- Patients were concurrently on medicine while administering ECT.

- Electrical dosage delivered during ECT was not considered during analysis.

\section{References}

[1]. Gallegos J, Vaidya P, D'Agati D, et al. (June 2012). "Decreasing adverse outcomes of unmodified electroconvulsive therapy: suggestions and possibilities". J ECT 28 (2): 77-81. doi:10.1097/YCT.0b013e3182359314.PMID 22531198.

[2]. Lisanby SH, Maddox JH, Prudic J, Devanand DP, Sackeim HA (June 2000). "The effects of electroconvulsive therapy on memory of autobiographical and public events". Arch. Gen. Psychiatry 57 (6): 581-90.doi:10.1001/archpsyc.57.6.581. PMID 10839336.

[3]. Andre, Linda (2009). Doctors of Deception: What They Don't Want You to Know About Shock Treatment. Rutgers University Press. ISBN 978-0-8135-4441-0.

[4]. Sackeim HA, Prudic J, Fuller R, Keilp J, Lavori PW, Olfson M (January 2007). "The cognitive effects of electroconvulsive therapy in community settings". Neuropsychopharmacology 32 (1): 244-54.doi:10.1038/sj.npp.1301180. PMID 16936712. Available on http://www.ncbi.nlm.nih.gov/pubmed/16936712

[5]. Breggin Peter (2007). "ECT Damages the Brain: Disturbing News for Patients and Shock Doctors Alike".Ethical Human Psychology and Psychiatry. 9 (2): 83-86.doi:10.1891/152315007782021196. 
The effects of modified brief pulse bilateral electroconvulsive therapy (ECT) on disease profile ....

[6]. MacQueen G., et al. (2007). "The long-term impact of treatment with electroconvulsive therapy on discrete memory systems in patients with bipolar disorder". Journal of Psychiatry and Neuroscience 32 (4): 241-249.PMC 1911194. PMID 17653292.

[7]. Fink, M. (July-August 2007). "Complaints of loss of personal memories after electroconvulsive therapy: evidence of a somatoform disorder?". Psychosomatics 48(4): 290-93. doi:10.1176/appi.psy.48.4.290. PMID 17600164.

[8]. Coentre R, et al. (May-June 2009). "Electroconvulsive therapy: myths and evidences". Acta Medica Portuguesa22 (3): 27580. PMID 19686628.

[9]. Zyss T, et al. (Nov-Dec 2008). "Shock therapy in psychiatry--historical feature". Psychiatrica Polska 42 (6): 797818.PMID 19441660.

[10]. Faedda GL, et al. (March 2009). "The origins of electroconvulsive therapy: Prof. Bini's first report on ECT".Journal of affective disorders. Epub Ahead of Print (1-3): 12-5. doi:10.1016/j.jad.2009.01.023. PMID 19268370.

[11]. Van Herck E, et al. (2009). "Electroconvulsive therapy for patients with intracranial aneurysms: a case study and literature review". Dutch Journal of Psychiatry 51 (1): 43-51.

[12]. Cristancho MA, et al. (Dec 2008). "Uncommon but serious complications associated with electroconvulsive therapy: recognition and management for the clinician". Current psychiatry reports 10 (6): 474-80. doi:10.1007/s11920-008-0076-4. PMID 18980730.

[13]. Busnello JV, et al. (Dec 2008). "Peripheral nucleotide hydrolysis in rats submitted to a model of electroconvulsive therapy". Prog Neuro psycho pharmacol Biol Psychiatry. 32(8): 1829-33. doi:10.1016/j.pnpbp. 2008 .08.007.PMID 18775464.

[14]. Malekian A, et al. (Jun 2009). "Knowledge of attitude toward experience and satisfaction with electroconvulsive therapy in a sample of Iranian patients". Journal of ECT. 25(2): 106-12. doi:10.1097/YCT .0b013 e3181 8050dc.PMID 18708944.

[15]. Wilkins KM, et al. (March 2008). "Efficacy of electroconvulsive therapy in the treatment of nondepressed psychiatric illness in elderly patients: a review of the literature". Journal of geriatric psychiatry and neurology. 21(1): 311. doi:10.1177/0891988707311027.PMID 18287164.

[16]. V.N. Bagadia, P.V. Pradhan, Jyoti Doshi and Ravi Abhayankar. "Evaluation of cognitive effects of ECT". Indian J Psychiatry. 1981 Oct-Dec; 23(4): 324-329. PMCID:PMC3012902

[17]. Neera ghaziuddin, Donna laughrin, and Bruno giordani. " Cognitive side effects of electroconvulsive therapy in adolescents". Journal of Child and Adolescent Psychopharmacology. Winter 2000, 10(4): 269-276. Doi:10.1089/cap.2000.10.269

[18]. Adhikari SR, Pradhan SN, Sharma SC, Shrestha BR, Shrestha S, Tabedar S. "Diagnostic variability and therapeutic efficacy of ECT in a Nepalese sample.’Kathmandu Univ Med J (KUMJ). 2008 Jan-Mar;6(1):41-8.

[19]. Ng C, Schweitzer I, Alexopoulos P, Celi E, Wong L, Tuckwell V, Sergejew A, Tiller J. "Efficacy and cognitive effects of right unilateral electroconvulsive therapy.”JECT. 2000 Dec; 16(4):370-9.

[20]. Vineet Bharadwaj, Sandeep Grover, Subho Chakrabarti, Ajit Avasthi and Natasha Kate. "Clinical profile and outcome of bipolar disorder patients receiving electroconvulsive therapy: A study from north india."indian J Psychiatry. 2012 Jan-Mar; 54(1): 41-47. doi: 10.4103/0019-5545.94644 PMCID: PMC3339218

[21]. Iannis M Zervas, Christos Theleritis, Costantin R Soldatos. “Using ECT in schizophrenia: a review from a clinical perspective.”'The World Journal of Biological Psychiatry (Impact Factor: 2.38). 04/2011; 13(2):96-105. DOI:10.3109/15622975.2011.564653 .

[22]. Brandon S, Cowley P, McDonald C, Neville P, Palmer R, Wellstood-Eason S. Leicester ECT trial: results in schizophrenia. Br J Psychiatry. 1985 Feb;146:177-83.

\section{Tables:}

Young Mania Rating Scale score $(\mathrm{N}=22)$

Table 1

\begin{tabular}{|c|c|c|c|c|c|c|c|c|}
\hline Total score & Before & Post $\quad 1^{\text {st }}$ & Post last & After 1 month(D) & \multicolumn{4}{|c|}{ Paired t test (significant yes or no) } \\
\hline & & & & & $\mathrm{A} \& \mathrm{~B}$ & $\mathrm{A \& C}$ & A\&D & $C \& D$ \\
\hline Mean & 37 & 33 & 25 & 24 & yes & yes & yes & yes \\
\hline Median & 38 & 32.5 & 27 & 26 & & & & \\
\hline
\end{tabular}

Interpretation of results: ECT resulted in decrease in psychopathology in mania in study participants as per YMRS scale.

\section{Hamilton Depression Rating Scale scores $(\mathrm{N}=23)$ :}

Graph 2

Table 2

\begin{tabular}{|c|c|c|c|c|c|c|c|c|}
\hline & $\begin{array}{l}\text { Before } \\
\text { ECT(A) }\end{array}$ & $\begin{array}{ll}\text { Post } & 1^{\text {st }} \\
\operatorname{ECT}(B) & \end{array}$ & $\begin{array}{ll}\text { Post } & \text { last } \\
\text { ECT(C) } & \end{array}$ & $\begin{array}{ll}\text { After } & 1 \\
\text { month(D) }\end{array}$ & \multicolumn{4}{|c|}{$\begin{array}{l}\text { Paired t test } \\
\text { (significant: yes or no) }\end{array}$} \\
\hline Mean & 24.25 & 18.85 & 10.75 & 10.16 & yes & yes & yes & no \\
\hline
\end{tabular}

Interpretation of results: ECT resulted in decrease in psychopathology in depression in study participants as per HDRS scale.

\section{PANSS Scale scores $(\mathrm{N}=42)$}

Table 3

\begin{tabular}{|l|l|l|l|l|l|l|l|l|}
\hline & \multicolumn{3}{|c|}{ Average score } & \multicolumn{3}{l|}{$\begin{array}{l}\text { Paired t test } \\
\text { (significant: Y/N) }\end{array}$} \\
\hline Item & (A) & (B) & (C) & (D) & A\&B & A\&C & A\&D & C\&D \\
\hline Positive Symptoms & 24.5 & 23.14 & 17.0 & 17.32 & yes & yes & yes & No \\
\hline Negative ymptoms & 18.73 & 18.31 & 16.82 & 16.58 & yes & yes & yes & No \\
\hline $\begin{array}{l}\text { General Psycho } \\
\text { Pathology }\end{array}$ & 35.68 & 33.36 & 29.07 & 28 & yes & yes & yes & yes \\
\hline Mean & 77.69 & 75.28 & 63.76 & 62 & yes & yes & yes & yes \\
\hline Median & 83 & 76.5 & 58 & 57 & & \multicolumn{5}{l}{} \\
\hline
\end{tabular}


The effects of modified brief pulse bilateral electroconvulsive therapy (ECT) on disease profile ....

\section{ACE-R scale scores}

Table 4

\begin{tabular}{|c|c|c|c|c|c|c|c|c|}
\hline Illness & \multicolumn{4}{|c|}{ ACE-R scale scores } & \multicolumn{4}{|c|}{$\begin{array}{l}\text { Paired t test } \\
\text { (significant: yes or no }\end{array}$} \\
\hline Orientation \& attention & 13.68 & 13.27 & 13.22 & 14.55 & no & no & yes & yes \\
\hline Memory & 18.90 & 17.45 & 16.40 & 19.95 & yes & yes & no & yes \\
\hline Fluency & 11.71 & 11.85 & 11.71 & 12.05 & no & no & no & no \\
\hline Mean & 73.57 & 73.38 & 72.28 & 79.98 & no & no & yes & yes \\
\hline \multicolumn{9}{|l|}{ Depressive disorder } \\
\hline Orientation \& attention & 15.82 & 15.91 & 15.21 & 15.8 & no & no & no & yes \\
\hline Memory & 20.71 & 20.78 & 19.34 & 21.45 & no & no & no & yes \\
\hline Fluency & 12.2 & 11.85 & 12.65 & 12.5 & no & no & no & no \\
\hline Language & 20.04 & 20.86 & 21.43 & 21.9 & no & yes & yes & no \\
\hline Memory & 14.07 & 14.45 & 16.33 & 17.56 & no & yes & yes & no \\
\hline Fluency & 7.42 & 7.11 & 7.19 & 7.14 & yes & yes & yes & no \\
\hline Language & 16.45 & 17.80 & 17.88 & 18.20 & yes & yes & yes & yes \\
\hline Visuospatial & 9.35 & 10.01 & 12.42 & 12.52 & no & yes & yes & no \\
\hline Mean & 60.71 & 61.92 & 68.95 & 70.52 & yes & yes & yes & yes \\
\hline
\end{tabular}

Table 5

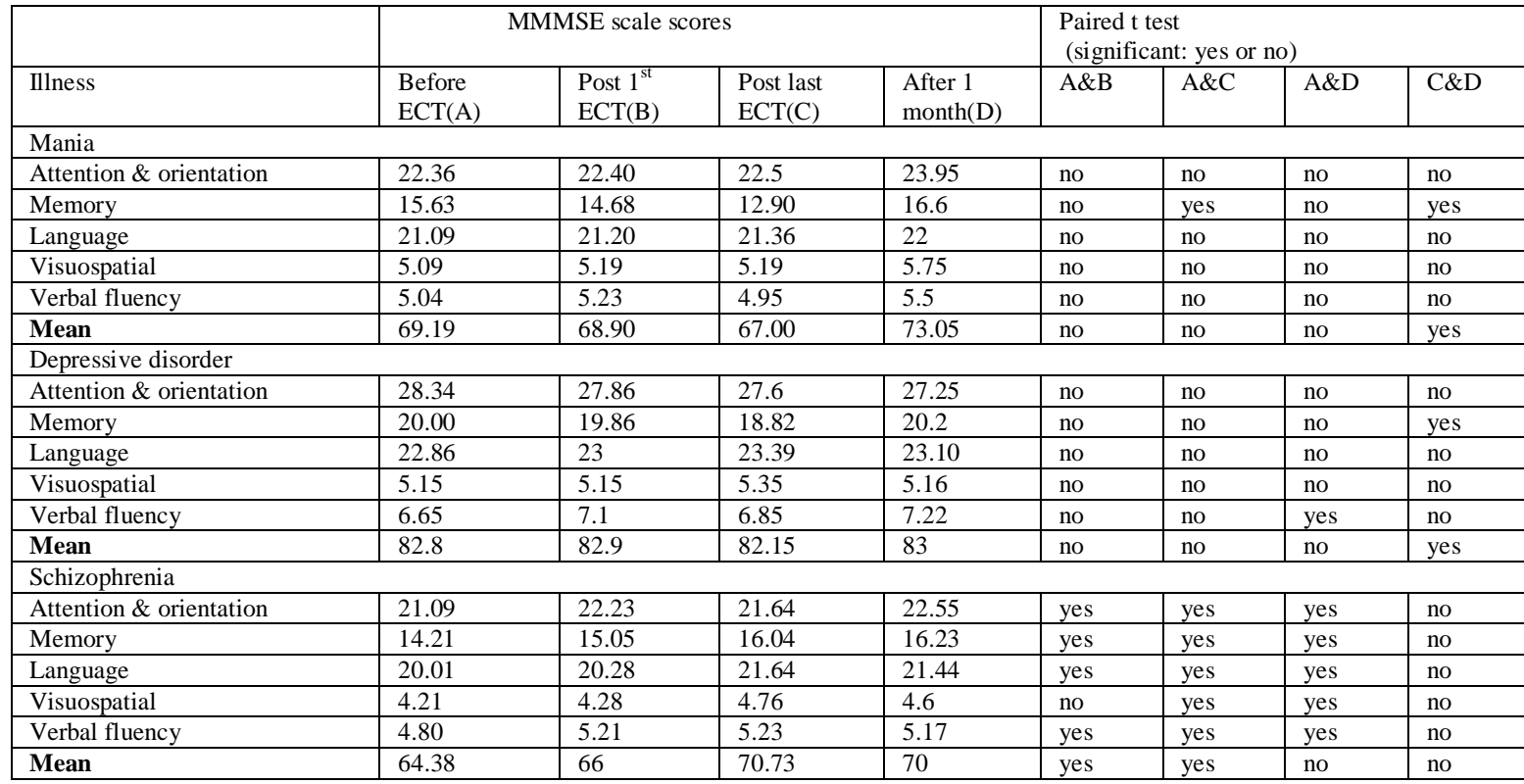

Table 6

\begin{tabular}{|c|c|c|c|c|c|c|c|c|}
\hline & \multicolumn{4}{|c|}{ PGI Memory scale scores } & \multicolumn{4}{|c|}{$\begin{array}{l}\text { Paired t test } \\
\text { (significant: yes or no) }\end{array}$} \\
\hline illness & $\begin{array}{l}\text { Before } \\
\text { ECT(A) }\end{array}$ & $\begin{array}{l}\text { Post } 1^{\text {st }} \\
\text { ECT(B) }\end{array}$ & Post last ECT(C) & $\begin{array}{l}\text { After 1 } \\
\text { month(D) }\end{array}$ & $\mathrm{A} \& \mathrm{~B}$ & $\mathrm{~A} \& \mathrm{C}$ & $\mathrm{A} \& \mathrm{D}$ & $\mathrm{C} \& \mathrm{D}$ \\
\hline mania & 59.76 & 56.47 & 54.28 & 63.3 & yes & yes & no & yes \\
\hline depressive disorder & 70.75 & 69.85 & 69.85 & 71.33 & no & no & no & yes \\
\hline schizophrenia & 58.38 & 58.71 & 62.19 & 62.48 & no & yes & yes & yes \\
\hline
\end{tabular}

